\title{
On Lie nilpotent rings and Cohen's Theorem
}

\author{
Jenő Szigeti and Leon van Wyk
}

\begin{abstract}
We study certain (two-sided) nil ideals and nilpotent ideals in a Lie nilpotent ring $R$. Our results lead us to showing that the prime radical $\operatorname{rad}(R)$ of $R$ comprises the nilpotent elements of $R$, and that if $L$ is a left ideal of $R$, then $L+\operatorname{rad}(R)$ is a two-sided ideal of $R$. This in turn leads to a Lie nilpotent version of Cohen's theorem, namely if $R$ is a Lie nilpotent ring and every prime (two-sided) ideal of $R$ is finitely generated as a left ideal, then every left ideal of $R$ containing the prime radical of $R$ is finitely generated (as a left ideal). For an arbitrary ring $R$ with identity we also consider its so-called $n$-th Lie center $Z_{n}(R), n \geq 1$, which is a Lie nilpotent ring of index $n$. We prove that if $C$ is a commutative submonoid of the multiplicative monoid of $R$, then the subring $\left\langle\mathrm{Z}_{n}(R) \cup C\right\rangle$ of $R$ generated by the subset $\mathrm{Z}_{n}(R) \cup C$ of $R$ is also Lie nilpotent of index $n$.
\end{abstract}

\section{INTRODUCTION}

One of the inspirations for this paper was a search for a Lie nilpotent version of a well known theorem in commutative algebra, namely Cohen's Theorem (see [Co]), which states that a commutative ring $R$ is Noetherian (i.e. every ideal of $R$ is finitely generated) if every prime ideal of $R$ is finitely generated.

Cohen's Theorem and Kaplansky's Theorem (see [Ka]) are quite often mentioned in the same breath. The latter states that a commutative Noetherian ring $R$ is a principal ideal ring (i.e. every left ideal of $R$ is principal and every right ideal of $R$ is principal) if and only if every maximal ideal of $R$ is principal. In fact, in [Re], an excellent paper which contains a thorough survey (including an extensive list of references) of quite a number of versions of Cohen's Theorem in various contexts, mention is made of the combined Kaplansky-Cohen Theorem, which states that a commutative ring $R$ is a principal ideal ring if and only if every prime ideal of $R$ is principal.

1991 Mathematics Subject Classification. 16D25, 16P40, 16U70, 16U80.

Key words and phrases. Lie nilpotent ring, Prime radical, Cohen's theorem, n-th Lie center.

The first author was supported by OTKA K-101515 of Hungary and by the TAMOP-4.2.1.B10/2/KONV-2010-0001 project with support by the European Union, co-financed by the European Social Fund.

The second author was supported by the National Research Foundation of South Africa under Grant No. UID 72375. Any opinion, findings and conclusions or recommendations expressed in this material are those of the authors and therefore the National Research Foundation does not accept any liability in regard thereto.

The authors thank P. N. Anh, L. Marki and J. H. Meyer for fruitful consultations. 
Moreover, Cohen's Theorem and the Kaplansky-Cohen Theorem are strengthened in [Re], and some generalizations of Cohen's Theorem, for example, Koh's (see $[\mathrm{Ko}]$ ) and Chandran's (see $[\mathrm{Ch}]$ ), are implied by results in [Re]. Other papers on Cohen's Theorem, some of of which contain module versions of Cohen's Theorem, include $[\mathrm{H}],[\mathrm{Jo}],[\mathrm{L}],[\mathrm{Na}]$ and $[\mathrm{Ni}]$.

In Section 2 we deal with certain products in a Lie nilpotent ring $R$ of index $n \geq 2$. We shall make use of classical results due to Jennings (see [Je]), and in order to ease readability, we provide short self-contained proofs of these theorems. A basic example of a Lie nilpotent ring $R$ of index $n \geq 2$ is also presented.

In Section 3 we show, amongst others, that the prime $\operatorname{radical} \operatorname{rad}(R)$ of a Lie nilpotent ring $R$ of index $n \geq 2$ comprises the nilpotent elements of $R$, and that if $L$ is a left ideal of $R$, then $L+\operatorname{rad}(R)$ is a two-sided ideal of $R$. This in turn leads to Theorem 3.3: if $R$ is a Lie nilpotent ring and every prime (two-sided) ideal of $R$ is finitely generated as a left ideal, then every left ideal of $R$ containing the prime radical of $R$ is finitely generated (as a left ideal). In this regard we mention (see $[\mathrm{Re}]$ and $[\mathrm{Kr}]$ ) that G. Michler and L. Small proved independently that a left fully bounded ring (such as a polynomial identity ring) in which every prime ideal is finitely generated as a left ideal is left noetherian. Therefore, although Theorem 3.3 is not new, the particular techniques employed in the present paper in the study of Lie nilpotent rings are important in their own right.

The authors note that they use the notation $\operatorname{rad}(R)$ for the prime (or lower nil) radical and $\mathrm{J}(R)$ for the Jacobson radical of $R$.

Another inspiration for this paper was Lemma 2.1 of [Sz], which plays a crucial role in the development of the Lie nilpotent determinant theory in [Sz] and [SzvW]. For an arbitrary ring $R$ with identity we consider in Section 4 its so-called $n$-th Lie center $\mathrm{Z}_{n}(R), n \geq 1$, which is a Lie nilpotent ring of index $n$. We prove the following broad generalization of the mentioned lemma: if $C$ is a commutative submonoid of the multiplicative monoid of $R$, then the subring $\left\langle\mathrm{Z}_{n}(R) \cup C\right\rangle$ of $R$ generated by the subset $\mathrm{Z}_{n}(R) \cup C$ of $R$ is also Lie nilpotent of index $n$.

\section{PRODUCTS IN LIE NILPOTENT RINGS}

Let $R$ be a ring, and let $[x, y]=x y-y x$ denote the additive commutator of the elements $x, y \in R$. It is well known that $(R,+,[]$,$) is a Lie ring and the following$ identities hold:

$$
\begin{gathered}
{[y, x]=-[x, y]} \\
{[[x, y], z]+[[y, z], x]+[[z, x], y]=0 \text { (Jacobian identity), }} \\
{[u v, x]=[u, v x]+[v, x u]=u[v, x]+[u, x] v .}
\end{gathered}
$$

The use of $[x, u v]=u[x, v]+[x, u] v$ and $[u v, x]=u[v, x]+[u, x] v$ gives that

$$
\begin{gathered}
{[[a, b y], x]=[b[a, y]+[a, b] y, x]=[b[a, y], x]+[[a, b] y, x]=} \\
b[[a, y], x]+[b, x][a, y]+[a, b][y, x]+[[a, b], x] y .
\end{gathered}
$$

For a sequence $x_{1}, x_{2}, \ldots, x_{n}$ of elements in $R$ we use the notation $\left[x_{1}, x_{2}, \ldots, x_{n}\right]_{n}^{*}$ for the left normed commutator (Lie-)product:

$$
\left[x_{1}\right]_{1}^{*}=x_{1} \text { and }\left[x_{1}, x_{2}, \ldots, x_{n}\right]_{n}^{*}=\left[\ldots\left[\left[x_{1}, x_{2}\right], x_{3}\right], \ldots, x_{n}\right] .
$$

Clearly, we have

$$
\left[x_{1}, x_{2}, \ldots, x_{n}, x_{n+1}\right]_{n+1}^{*}=\left[\left[x_{1}, x_{2}, \ldots, x_{n}\right]_{n}^{*}, x_{n+1}\right]=\left[\left[x_{1}, x_{2}\right], x_{3}, \ldots, x_{n}, x_{n+1}\right]_{n}^{*} .
$$


A ring $R$ is called Lie nilpotent of index $n$ (or having property $\mathrm{L}_{n}$ ) if

$$
\left[x_{1}, x_{2}, \ldots, x_{n}, x_{n+1}\right]_{n+1}^{*}=0
$$

is a polynomial identity on $R$. If $R$ has property $\mathrm{L}_{n}$, then $\left[x_{1}, x_{2}, \ldots, x_{n}\right]_{n}^{*} \in \mathrm{Z}(R)$ is central for all $x_{1}, x_{2}, \ldots, x_{n} \in R$.

Let $k_{0}=0$ and $1 \leq k_{1}, \ldots, k_{n}, k_{n+1} \leq m$ be integers such that $k_{1}+\cdots+k_{n}+$ $k_{n+1}=m$ and let $K$ be a field. The pair $(i, j)$ of integers satisfies $(*)$ if

$$
k_{0}+k_{1}+\cdots+k_{t-1}<i \leq k_{0}+k_{1}+\cdots+k_{t}<j \leq m
$$

for some (unique) index $1 \leq t \leq n$. One of the basic examples of a $K$-algebra satisfying $\mathrm{L}_{n}$ is the $K$-subalgebra

$$
R=R_{m}\left(k_{1}, \ldots, k_{n}, k_{n+1}\right)=\left\{\sum a_{i, j} E_{i, j} \mid a_{i, j} \in K \text { and }(i, j) \text { satisfies }(*)\right\}
$$

of block upper triangular $m \times m$ matrices of the full matrix algebra $\mathrm{M}_{m}(K)$, where $E_{i, j}$ is the standard matrix unit with 1 in the $(i, j)$ position. Clearly, the ordinary nilpotency $R^{n+1}=\{0\} \neq R^{n}$ implies $\mathrm{L}_{n}$, and the addition of the center (scalar matrices) yields a unitary subring $R+K I_{m}$ of $\mathrm{M}_{m}(K)$ also with $\mathrm{L}_{n}$. The $K$ dimension of $R+K I_{m}$ is

$$
\operatorname{dim}_{K}(R)=1+\frac{1}{2}\left(m^{2}-k_{1}^{2}-\cdots-k_{n}^{2}-k_{n+1}^{2}\right) .
$$

Conjecture. If $S$ is a (unitary) $K$-subalgebra of $\mathrm{M}_{m}(K)$ with $L_{n}$ and $n+1 \leq m$, then

$$
\operatorname{dim}_{K}(S) \leq 1+\frac{1}{2}\left(m^{2}-k_{1}^{2}-\cdots-k_{n}^{2}-k_{n+1}^{2}\right)
$$

for some integers $1 \leq k_{1}, \ldots, k_{n}, k_{n+1} \leq m$ with $k_{1}+\cdots+k_{n}+k_{n+1}=m$.

For $n=1$ our conjecture becomes a classical theorem of Schur about the maximal dimension of a commutative subalgebra of $\mathrm{M}_{m}(K)$.

We call a ring $R$ Lie nilpotent if it is Lie nilpotent of index $n$ for some $n \geq 1$ (see, for example, [D], [DF], [Je] and [Ro]). Notice that $\mathrm{M}_{2}(K)$ is not Lie nilpotent, i.e. it does not satisfy $\mathrm{L}_{n}$ for any $n \geq 1$.

2.1.Proposition. Let $R$ be a ring with $\mathrm{L}_{2}$ and $a, b, c, a_{0}, a_{1}, \ldots, a_{k} \in R$.

(1) $[a, b][a, c]=0$.

(2) If $a b=0$, then bxbya $=0$ for all $x, y \in R$ (i.e. $b R b R a=\{0\}$ ).

(3) If $a_{0} a_{1} \cdots a_{k}=0$, then

$$
a_{1} x_{1} a_{1} y_{1} a_{2} x_{2} a_{2} y_{2} \cdots a_{k} x_{k} a_{k} y_{k} a_{0}=0
$$

for all $x_{i}, y_{i} \in R, 1 \leq i \leq k$.

\section{Proof.}

(1): Take $x=c$ and $y=a$ in

$$
[[a, b y], x]=b[[a, y], x]+[b, x][a, y]+[a, b][y, x]+[[a, b], x] y .
$$

(2): $a b=0$ and the $\mathrm{L}_{2}$ property of $R$ imply that bya $=[b y, a]$ is central, whence

$$
\text { bxbya }=(\text { bya }) b x=0
$$

follows.

(3): In order to see the validity of the implication for $k=1$, take $a=a_{0}$ and $b=a_{1}$ in part (2). In the next step of the induction we assume that our statement 
holds for some $k \geq 1$ and consider the product $a_{0} a_{1} \cdots a_{k} a_{k+1}=0$ in $R$. Using the induction hypothesis for $\left(a_{0} a_{1}\right) a_{2} \cdots a_{k} a_{k+1}=0$, we obtain that

$$
a_{2} x_{2} a_{2} y_{2} a_{3} \cdots a_{k} x_{k} a_{k} y_{k} a_{k+1} x_{k+1} a_{k+1} y_{k+1}\left(a_{0} a_{1}\right)=0
$$

for all $x_{i}, y_{i} \in R, 2 \leq i \leq k+1$. Now the choice of

$$
a=a_{2} x_{2} a_{2} y_{2} a_{3} \cdots a_{k} x_{k} a_{k} y_{k} a_{k+1} x_{k+1} a_{k+1} y_{k+1} a_{0} \text { and } b=a_{1}
$$

in part (2) gives that

$$
0=b x_{1} b y_{1} a=a_{1} x_{1} a_{1} y_{1} a_{2} x_{2} a_{2} y_{2} a_{3} \cdots a_{k} x_{k} a_{k} y_{k} a_{k+1} x_{k+1} a_{k+1} y_{k+1} a_{0}
$$

for all $x_{i}, y_{i} \in R, 1 \leq i \leq k+1$. It follows that our statement is valid for $k+1$.

2.2. Theorem ([Je]). Let $n \geq 3$ be an integer and $R$ be a ring with $\mathrm{L}_{n}$. Then

$$
\left[x_{1}, x_{2}, \ldots, x_{n}\right]_{n}^{*} \cdot\left[y_{1}, y_{2}, \ldots, y_{n}\right]_{n}^{*}=0
$$

for all $x_{i}, y_{i} \in R, 1 \leq i \leq n$. Thus the two sided ideal

$N=R\left\{\left[x_{1}, x_{2}, \ldots, x_{n}\right]_{n}^{*} \mid x_{i} \in R, 1 \leq i \leq n\right\}=\left\{\left[x_{1}, x_{2}, \ldots, x_{n}\right]_{n}^{*} \mid x_{i} \in R, 1 \leq i \leq n\right\} R$ generated by the (central) elements $\left[x_{1}, x_{2}, \ldots, x_{n}\right]_{n}^{*}$ is nilpotent with $N^{2}=\{0\}$.

Proof. Take

$$
x=\left[x_{1}, x_{2}, \ldots, x_{n-2}\right]_{n-2}^{*}, y=x_{n-1} \text { and } z=\left[y_{1}, y_{2}, \ldots, y_{n-1}\right]_{n-1}^{*}
$$

in the Jacobi identity

$$
[[x, y], z]+[[y, z], x]+[[z, x], y]=0 \text {. }
$$

Since

$[[y, z], x]=-[[z, y], x]=-\left[\left[\left[y_{1}, y_{2}, \ldots, y_{n-1}\right]_{n-1}^{*}, y\right], x\right]=-\left[y_{1}, y_{2}, \ldots, y_{n-1}, y, x\right]_{n+1}^{*}=0$ and

$$
[[z, x], y]=\left[\left[\left[y_{1}, y_{2}, \ldots, y_{n-1}\right]_{n-1}^{*}, x\right], y\right]=\left[y_{1}, y_{2}, \ldots, y_{n-1}, x, y\right]_{n+1}^{*}=0
$$

are consequences of the $\mathrm{L}_{n}$ property, we obtain that

$$
\begin{gathered}
0=[[x, y], z]=\left[\left[\left[x_{1}, x_{2}, \ldots, x_{n-2}\right]_{n-2}^{*}, x_{n-1}\right],\left[y_{1}, y_{2}, \ldots, y_{n-1}\right]_{n-1}^{*}\right]= \\
{\left[\left[x_{1}, x_{2}, \ldots, x_{n-1}\right]_{n-1}^{*},\left[y_{1}, y_{2}, \ldots, y_{n-1}\right]_{n-1}^{*}\right] .}
\end{gathered}
$$

Now take

$$
a=\left[y_{1}, y_{2}, \ldots, y_{n-1}\right]_{n-1}^{*}, b=\left[x_{1}, x_{2}, \ldots, x_{n-1}\right]_{n-1}^{*}, x=x_{n} \text { and } y=y_{n}
$$

in

$$
[[a, b y], x]=b[[a, y], x]+[b, x][a, y]+[a, b][y, x]+[[a, b], x] y .
$$

Since

$$
\begin{gathered}
{[[a, b y], x]=\left[\left[\left[y_{1}, y_{2}, \ldots, y_{n-1}\right]_{n-1}^{*}, b y\right], x\right]=\left[y_{1}, y_{2}, \ldots, y_{n-1}, b y, x\right]_{n+1}^{*}=0,} \\
{[[a, y], x]=\left[\left[\left[y_{1}, y_{2}, \ldots, y_{n-1}\right]_{n-1}^{*}, y\right], x\right]=\left[y_{1}, y_{2}, \ldots, y_{n-1}, y, x\right]_{n+1}^{*}=0,} \\
{[[a, b], x]=\left[\left[\left[y_{1}, y_{2}, \ldots, y_{n-1}\right]_{n-1}^{*}, b\right], x\right]=\left[y_{1}, y_{2}, \ldots, y_{n-1}, b, x\right]_{n+1}^{*}=0}
\end{gathered}
$$

are consequences of the $\mathrm{L}_{n}$ property and

$$
[a, b]=\left[\left[y_{1}, y_{2}, \ldots, y_{n-1}\right]_{n-1}^{*},\left[x_{1}, x_{2}, \ldots, x_{n-1}\right]_{n-1}^{*}\right]=0,
$$

we obtain that

$$
0=[b, x][a, y]=\left[\left[x_{1}, x_{2}, \ldots, x_{n-1}\right]_{n-1}^{*}, x_{n}\right]\left[\left[y_{1}, y_{2}, \ldots, y_{n-1}\right]_{n-1}^{*}, y_{n}\right]=
$$




$$
\left[x_{1}, x_{2}, \ldots, x_{n}\right]_{n}^{*}\left[y_{1}, y_{2}, \ldots, y_{n}\right]_{n}^{*} .
$$

2.3.Remark. The $m$-generated $(m \geq 4)$ Grassmann algebra

$$
\left.E^{(m)}=K\left\langle v_{1}, \ldots, v_{m}\right| v_{i} v_{j}+v_{j} v_{i}=0 \text { for all } 1 \leq i \leq j \leq m\right\rangle
$$

over a field $K$ (with $4 \neq 0$ ) has property $\mathrm{L}_{2}$ and

$$
\left[v_{1}, v_{2}\right]_{2}^{*} \cdot\left[v_{3}, v_{4}\right]_{2}^{*}=\left[v_{1}, v_{2}\right] \cdot\left[v_{3}, v_{4}\right]=4 v_{1} v_{2} v_{3} v_{4} \neq 0
$$

shows that Theorem 2.2 is not valid for $n=2$. The fact that $a$ occurs twice in $[a, b][a, c]$ is essential in part (1) of Proposition 2.1.

2.4. Corollary ([Je]). Let $n \geq 2$ be an integer and $R$ be a ring with $\mathrm{L}_{n}$.

(1) The ideal

$$
I(2)=R[R, R] R=\left\{\sum_{1 \leq i \leq t} r_{i}\left[a_{i}, b_{i}\right] s_{i} \mid r_{i}, a_{i}, b_{i}, s_{i} \in R, 1 \leq i \leq t\right\} \triangleleft R
$$

is nil.

(2) The ideal

$$
\begin{gathered}
I(3)=R[[R, R], R] R=\left\{\sum_{1 \leq i \leq t} r_{i}\left[\left[a_{i}, b_{i}\right], c_{i}\right] s_{i} \mid r_{i}, a_{i}, b_{i}, c_{i}, s_{i} \in R, 1 \leq i \leq t\right\} \triangleleft R \\
\quad \text { is nilpotent of index } 2^{n-2} .
\end{gathered}
$$

Proof. In both cases we use the ideal $N \triangleleft R$ generated by the (central) elements $\left[x_{1}, x_{2}, \ldots, x_{n}\right]_{n}^{*}$ and apply an induction with respect to $n$.

(1): If $n=2$, then part (1) of Proposition 2.1 gives that $\left[a_{i}, b_{i}\right]^{2}=0$ for each $1 \leq i \leq t$. Thus

$$
\left(\sum_{1 \leq i \leq t} r_{i}\left[a_{i}, b_{i}\right] s_{i}\right)^{t+1}=0
$$

follows from the centrality of the $\left[a_{i}, b_{i}\right]$ 's. Now assume that $(1)$ is valid in any ring with $\mathrm{L}_{n-1}$ (for some $n \geq 3$ ) and consider an element

$$
\sum_{1 \leq i \leq t} r_{i}\left[a_{i}, b_{i}\right] s_{i} \in R[R, R] R
$$

in a ring $R$ with $\mathrm{L}_{n}$. Since the factor $\operatorname{ring} \bar{R}=R / N$ satisfies $\mathrm{L}_{n-1}$, the induction hypothesis gives that for some $k \geq 1$ we have

$$
\left(\sum_{1 \leq i \leq t} \overline{r_{i}}\left[\overline{a_{i}}, \overline{b_{i}}\right] \overline{s_{i}}\right)^{k}=\overline{0}
$$

in $\bar{R}$. Since $N^{2}=\{0\}$ by Theorem 2.2 , we have

$$
\left(\sum_{1 \leq i \leq t} r_{i}\left[a_{i}, b_{i}\right] s_{i}\right)^{2 k}=0
$$

in $R$.

(2): If $n=2$, then $R[[R, R], R] R=\{0\}$. Now assume that (2) is valid in any ring with $\mathrm{L}_{n-1}(n \geq 3)$ and consider a sequence of elements $\left[\left[a_{i}, b_{i}\right], c_{i}\right], 1 \leq i \leq q=2^{n-2}$ in $R[[R, R], R] R$, where $R$ is a ring with $\mathrm{L}_{n}$. Since the factor $\operatorname{ring} \bar{R}=R / N$ satisfies $\mathrm{L}_{n-1}$, the induction hypothesis gives that for $p=2^{n-3}$

$$
\left[\left[\overline{a_{1}}, \overline{b_{1}}\right], \overline{c_{1}}\right] \bar{R}\left[\left[\overline{a_{2}}, \overline{b_{2}}\right], \overline{c_{2}}\right] \bar{R} \cdots \bar{R}\left[\left[\overline{a_{p}}, \overline{b_{p}}\right], \overline{c_{p}}\right]=\{\overline{0}\}
$$

holds in $\bar{R}$. Thus

$$
\left[\left[a_{1}, b_{1}\right], c_{1}\right] R\left[\left[a_{2}, b_{2}\right], c_{2}\right] R \cdots R\left[\left[a_{p}, b_{p}\right], c_{p}\right] \subseteq N
$$

and similarly

$$
\left[\left[a_{p+1}, b_{p+1}\right], c_{p+1}\right] R\left[\left[a_{p+2}, b_{p+2}\right], c_{p+2}\right] R \cdots R\left[\left[a_{p+p}, b_{p+p}\right], c_{p+p}\right] \subseteq N
$$


for all $a_{i}, b_{i}, c_{i} \in R, 1 \leq i \leq 2 p=q$. It follows that

$$
\left[\left[a_{1}, b_{1}\right], c_{1}\right] R \cdots R\left[\left[a_{p}, b_{p}\right], c_{p}\right] R\left[\left[a_{p+1}, b_{p+1}\right], c_{p+1}\right] R \cdots R\left[\left[a_{p+p}, b_{p+p}\right], c_{p+p}\right] \subseteq N^{2} .
$$

In view of Theorem 2.2 , we obtain that

$$
(R[[R, R], R] R)^{q} \subseteq N^{2}=\{0\} .
$$

2.5.Theorem. Let $n \geq 2$ be an integer and $R$ be a ring with $\mathrm{L}_{n}$. If $a, b \in R$, $a b=0$ and $q=q(n)=2^{n-2}$, then

$$
b x_{1} b y_{1} a z_{1} b x_{2} b y_{2} a z_{2} \cdots z_{q-1} b x_{q} b y_{q} a=0 \text {, i.e. } \underbrace{b R b R a}_{1 .} R \underbrace{b R b R a}_{2 .} R \ldots R \underbrace{b R b R a}_{q .}=\{0\}
$$

for all $x_{i}, y_{i}, z_{i} \in R, 1 \leq i \leq q\left(z_{q}=1\right)$.

Proof. If $n=2$, then $q(2)=1$ and part (2) of Proposition 2.1 gives the result. Now assume (by induction) that our statement is valid in any ring with $\mathrm{L}_{n-1}$ (for some $n \geq 3$ ) and consider the elements $a, b \in R$ with $a b=0$ in a ring $R$ with $\mathrm{L}_{n}$. In view of Theorem 2.2, we have $N^{2}=\{0\}$ for the ideal $N \triangleleft R$ generated by the (central) elements $\left[x_{1}, x_{2}, \ldots, x_{n}\right]_{n}^{*}$. Since the factor $\operatorname{ring} R / N$ satisfies $\mathrm{L}_{n-1}$, the induction hypothesis gives that

$$
\begin{gathered}
(b+N)\left(x_{1}+N\right)(b+N)\left(y_{1}+N\right)(a+N)\left(z_{1}+N\right) \cdots \\
\cdots\left(z_{q(n-1)-1}+N\right)(b+N)\left(x_{q(n-1)}+N\right)(b+N)\left(y_{q(n-1)}+N\right)(a+N)=0+N
\end{gathered}
$$

in $R / N$. Thus

$$
b x_{1} b y_{1} a z_{1} \cdots z_{q(n-1)-1} b x_{q(n-1)} b y_{q(n-1)} a \in N
$$

and similarly

$$
b x_{q(n-1)+1} b y_{q(n-1)+1} a z_{q(n-1)+1} \cdots z_{q(n-1)+q(n-1)-1} b x_{q(n-1)+q(n-1)} b y_{q(n-1)+q(n-1)} a \in N
$$

for all $x_{i}, y_{i} \in R, 1 \leq i \leq 2 q(n-1)$ and $z_{i} \in R, 1 \leq i \leq 2 q(n-1)-1, i \neq q(n-1)$. Now for any $z_{q(n-1)} \in R$ we have

$$
\begin{gathered}
\left(b x_{1} b y_{1} a z_{1} \cdots z_{q(n-1)-1} b x_{q(n-1)} b y_{q(n-1)} a\right) z_{q(n-1)}\left(b x_{q(n-1)+1} b y_{q(n-1)+1} a z_{q(n-1)+1} \cdots\right. \\
\left.\cdots z_{q(n-1)+q(n-1)-1} b x_{q(n-1)+q(n-1)} b y_{q(n-1)+q(n-1)} a\right) \in N^{2}
\end{gathered}
$$

and $2 q(n-1)=q(n)$ proves that the statement is valid in $R$.

\section{COHEN'S THEOREM FOR LIE NILPOTENT RINGS}

3.1.Proposition. Let $R$ be a ring with $\mathrm{L}_{n}(n \geq 2)$ and $a, b \in R$.

(1) If $P \triangleleft R$ is a prime ideal and $a b \in P$, then $a \in P$ or $b \in P$. In other words: all prime ideals of $R$ are completely prime.

(2) The prime radical of $R$ is the set of all nilpotent elements:

$$
\operatorname{rad}(R)=\left\{u \in R \mid u^{k}=0 \text { for some } k \geq 1\right\} .
$$

(3) The factor ring $R / \operatorname{rad}(R)$ is commutative.

(4) If $L \leq_{l} R$ is a left ideal of $R$, then $L+\operatorname{rad}(R) \triangleleft R$ is a two sided ideal of $R$. In particular, all left ideals containing $\operatorname{rad}(R)$ are two sided ideals. 


\section{Proof.}

(1): The factor ring $S=R / P$ is a prime ring with $\mathrm{L}_{n}$ and $a b \in P$ implies that

$$
\bar{a} \bar{b}=(a+P)(b+P)=\overline{0}
$$

in $S$. The application of Theorem 2.5 gives that

$$
\underbrace{\bar{b} S \bar{b} S \bar{a}}_{1 .} S \underbrace{\bar{b} S \bar{b} S \bar{a}}_{2 .} S \ldots S \underbrace{\bar{b} S \bar{b} S \bar{a}}_{q .}=\{0\},
$$

where $q=q(n)=2^{n-2}$. Since $S$ is prime, we deduce that $\bar{a}=\overline{0}$ or $\bar{b}=\overline{0}$. Thus we have $a \in P$ or $b \in P$.

(2): Let $P \triangleleft R$ be an arbitrary prime ideal of $R$ and $u \in R$ a nilpotent element with $u^{k}=0$. Now the iterated application of part (1) of the present Proposition 3.1 gives that $u \in P$ and

$$
\left\{u \in R \mid u^{k}=0 \text { for some } k \geq 1\right\} \subseteq \operatorname{rad}(R) .
$$

The reverse containment holds in any ring.

(3): Part (1) of Corollary 2.4 gives that $[x, y] \in R[R, R] R$ is nilpotent, whence $[x, y] \in \operatorname{rad}(R)$ follows by part (2) of the present Proposition 3.1.

(4) Since the sum of left ideals is a left ideal, we only have to show that $(x+u) r \in L+\operatorname{rad}(R)$ for all $x \in L, u \in \operatorname{rad}(R)$ and $r \in R$. We have

$$
(x+u) r=r x+[x, r]+u r
$$

where $r x \in L, u r \in \operatorname{rad}(R)$ and the nilpotency of $[x, r]$ gives that $[x, r] \in \operatorname{rad}(R)$ as in part (3) above.

3.2.Remark. If $R$ is a simple (unitary) Lie nilpotent ring, then the two sided ideal $\operatorname{rad}(R)$ is zero and the commutativity of $R \cong R / \operatorname{rad}(R)$ implies that $R$ is a field.

We have now collected sufficient tools to obtain a Lie nilpotent version of the following famous theorem in commutative algebra:

Cohen's Theorem. If every prime ideal of a commutative ring $R$ is finitely generated, then every ideal of $R$ is finitely generated (i.e. $R$ is Noetherian).

In quite a number of proofs of some versions of Cohen's Theorem Zorn's Lemma comes in handy (see, for example $[\mathrm{S}]$ ), as is the case below:

3.3. Theorem. If the (completely) prime ideals of a Lie nilpotent ring $R$ are finitely generated as left ideals, then every (left) ideal of $R$ containing $\operatorname{rad}(R)$ is finitely generated as a left ideal.

Proof. For the sake of contradiction suppose that

$$
\mathcal{N}=\{L \subseteq R \mid L \text { is a non-finitely generated left ideal of } R \text { and } \operatorname{rad}(R) \subseteq L\}
$$

is a non-empty set. In view of part (4) of Proposition 3.1 any left ideal $L \subseteq R$ with $\operatorname{rad}(R) \subseteq L$ is a two sided ideal of $R$. Thus the elements of $\mathcal{N}$ are two sided ideals. A straigthforward argument shows that the union of the (left) ideals of a chain (with respect to the containment relation) in $\mathcal{N}$ is also an element of $\mathcal{N}$. The application of Zorn's lemma gives the existence of a maximal element $P$ in $\mathcal{N}$. We claim that $P$ is a completely prime ideal of $R$. 
Assume that $a b \in P$ and $a, b \in R \backslash P$. The maximality of $P$ and $P \subseteq P+R b$, $b \in P+R b$ imply that the (left) ideal

$$
P+R b=R\left(p_{1}+r_{1} b\right)+\cdots+R\left(p_{k}+r_{k} b\right)
$$

is finitely generated by some elements $p_{i}+r_{i} b, 1 \leq i \leq k$ with $p_{i} \in P$ and $r_{i} \in R$. Consider the set

$$
K=\{x \in R \mid x b \in P\} .
$$

Clearly, $K \subseteq R$ is a left ideal of $R$ and $a \in K$. The containment $P \subseteq K$ follows from the fact that $P$ is a two sided ideal. The maximality of $P$ ensures that $K$ is a finitely generated left ideal, whence we obtain that $K b \subseteq R$ is also a finitely generated left ideal. We claim that

$$
P=R p_{1}+\cdots+R p_{k}+K b .
$$

Since $p_{i} \in P$ and $K b \subseteq P$, we have $R p_{1}+\cdots+R p_{k}+K b \subseteq P$. On the other hand, an element $p \in P \subseteq P+R b$ can be written as

$$
p=s_{1}\left(p_{1}+r_{1} b\right)+\cdots+s_{k}\left(p_{k}+r_{k} b\right),
$$

whence

$$
\left(s_{1} r_{1}+\cdots+s_{k} r_{k}\right) b=p-s_{1} p_{1}-\cdots-s_{k} p_{k} \in P
$$

and $s_{1} r_{1}+\cdots+s_{k} r_{k} \in K$ follow. Thus we have

$$
p=\left(s_{1} p_{1}+\cdots+s_{k} p_{k}\right)+\left(s_{1} r_{1}+\cdots+s_{k} r_{k}\right) b \in R p_{1}+\cdots+R p_{k}+K b .
$$

and $P \subseteq R p_{1}+\cdots+R p_{k}+K b$.

Since $K b$ is a finitely generated left ideal, we obtain that $P=R p_{1}+\cdots+R p_{k}+$ $K b$ is also a finitely generated left ideal of $R$, a contradiction. Thus $a b \in P$ and $a, b \in R \backslash P$ is impossible, proving that $P$ is completely prime.

Now $P \in \mathcal{N}$ contradicts the condition that all completely prime ideals are finitely generated as left ideals. It follows that $\mathcal{N}=\varnothing$ and our proof is complete.

3.4.Remark. Since Theorem 3.3 concerns the left ideals containing the prime radical, it is not a full generalization of Cohen's Theorem. On the other hand in a certain sense Theorem 3.3 is stronger than the existing non-commutaive generalizations of Cohen's Theorem. The reason is that prime left ideals are not used, we impose conditions only on the two sided prime ideals.

3.5.Remark. Since in the Lie nilpotent case $\bar{R}=R / \operatorname{rad}(R)$ is commutative (see (3) of Proposition 3.1), a direct application of Cohen's original theorem to $\bar{R}$ gives the following weaker version of Theorem 3.3:

If the (completely) prime ideals and the prime radical $\operatorname{rad}(R)$ of a Lie nilpotent $R$ are finitely generated as left ideals, then every (left) ideal of $R$ containing $\operatorname{rad}(R)$ is finitely generated as a left ideal.

It seems that the containment of the prime radical cannot be omitted in this direct application.

\section{THE $n$-TH LIE CENTER}

Let $R$ be an arbitrary ring with 1 , the $n$-th Lie center of $R$ is defined as

$$
\mathrm{Z}_{n}(R)=\left\{r \in R \mid\left[r, x_{1}, \ldots, x_{n}\right]_{n+1}^{*}=0 \text { for all } x_{i} \in R, 1 \leq i \leq n\right\} .
$$


The fact that $\mathrm{Z}_{n}(R)$ is a (unitary) subring of $R$ is a consequence of $[r s, x]=[r, s x]+$ $[s, x r]$ and

$$
\mathrm{Z}(R)=\mathrm{Z}_{1}(R) \subseteq \mathrm{Z}_{2}(R) \subseteq \cdots \subseteq \mathrm{Z}_{n}(R) \subseteq \mathrm{Z}_{n+1}(R) \subseteq \cdots
$$

follows from

$$
\left[r, x_{1}, \ldots, x_{n}, x_{n+1}\right]_{n+2}^{*}=\left[\left[r, x_{1}, \ldots, x_{n}\right]_{n+1}^{*}, x_{n+1}\right]
$$

Since

$$
\left[[r, s], x_{1}, \ldots, x_{n}\right]_{n+1}^{*}=\left[\left[r, s, x_{1}, \ldots x_{n-1}\right]_{n+1}^{*}, x_{n}\right],
$$

$r \in \mathrm{Z}_{n}(R)$ implies $[r, s] \in \mathrm{Z}_{n}(R)$ for all $s \in R$, so that $\mathrm{Z}_{n}(R)$ is a Lie ideal.

In any Lie ring $\left[x_{1}, \ldots, x_{k}, r\right]_{k+1}^{*}$ can be written as a sum of $2^{k-1}$ elements of the form $\pm\left[r, x_{\pi(1)}, \ldots, x_{\pi(k)}\right]_{k+1}^{*}$, where $\pi$ is some permutation of $\{1,2, \ldots, k\}$ (the use of the Jacobi identity and an easy induction on $k$ works). It follows that $\left[x_{1}, \ldots, x_{k}, r, x_{k+1}, \ldots, x_{n}\right]_{n+1}^{*}$ can be written as a sum of some

$$
\pm\left[r, x_{\pi(1)}, \ldots, x_{\pi(k)}, x_{k+1}, \ldots, x_{n}\right]_{n+1}^{*} .
$$

Thus $r \in \mathrm{Z}_{n}(R)$ implies that $\left[x_{1}, \ldots, x_{k}, r, x_{k+1}, \ldots, x_{n}\right]_{n+1}^{*}=0$ for all $x_{i} \in R$, $1 \leq i \leq n$. Consider the elements $r=E_{2,3}$ and $y_{1}=E_{3,4}, y_{2}=E_{1,2}$ in the $K$ subalgebra $R=K I_{4}+R_{4}(1,1,1,1)$ of $\mathrm{M}_{4}(K)$ (see the example in Section 2). For $x_{1}, x_{2} \in R$ we have $\alpha, \beta, \gamma \in K$ such that $\left[x_{1}, x_{2}\right]=\alpha E_{1,3}+\beta E_{1,4}+\gamma E_{2,4}$. Thus

$$
\left[\left[x_{1}, x_{2}\right], r\right]=0 \text { and }\left[\left[r, y_{1}\right], y_{2}\right]=-E_{1,4} \neq 0
$$

show that the implication

$$
\left[\left[x_{1}, x_{2}\right], r\right]=0 \text { for all } x_{1}, x_{2} \in R \Longrightarrow r \in \mathrm{Z}_{2}(R)
$$

(the converse of the mentioned one) is not valid.

The ring $\mathrm{Z}_{n}(R)$ obviously has the $\mathrm{L}_{n}$ property, a much stronger statement is the following.

4.1. Theorem. Let $n \geq 1$ be an integer and $C \subseteq R$ a commutative submonoid of the multiplicative monoid of $R$. Then the subring $S=\left\langle\mathrm{Z}_{n}(R) \cup C\right\rangle$ of $R$ generated by the subset $\mathrm{Z}_{n}(R) \cup C \subseteq R$ also has the $\mathrm{L}_{n}$ property, i.e.

$$
\left[x_{1}, x_{2}, \ldots, x_{n}, x_{n+1}\right]_{n+1}^{*}=0
$$

is a polynomial identity on $S$.

Proof. Since $c r=r c-[r, c]$ and $[r, c] \in \mathrm{Z}_{n}(R)$ for all $r \in \mathrm{Z}_{n}(R)$ and $c \in C$, we deduce that any element of the subring $S=\left\langle\mathrm{Z}_{n}(R) \cup C\right\rangle$ can be written as

$$
r_{1} c_{1}+\cdots+r_{t} c_{t}
$$

with $r_{1}, \ldots, r_{t} \in \mathrm{Z}_{n}(R)$ and $c_{1}, \ldots, c_{t} \in C$ (notice that $1 \in \mathrm{Z}_{n}(R) \cap C$ ).

In order to check that $\left[x_{1}, x_{2}, \ldots, x_{n}, x_{n+1}\right]_{n+1}^{*}=0$ is a polynomial identity on $S$, it is enough to consider substitutions of the form

$$
x_{1}=r_{1} c_{1}, \ldots, x_{n}=r_{n} c_{n}, x_{n+1}=r_{n+1} c_{n+1}
$$

with $r_{i} \in \mathrm{Z}_{n}(R)$ and $c_{i} \in C(1 \leq i \leq n+1)$.

If $1 \leq k \leq n$, then we claim that

$$
\begin{gathered}
{\left[r_{1} c_{1}, \ldots, r_{n} c_{n}, r_{n+1} c_{n+1}\right]_{n+1}^{*}=} \\
r_{n+1}\left[r_{n}\left[r_{n-1}\left[\ldots r_{k+2}\left[r_{k+1}\left[\left[r_{1} c_{1}, \ldots, r_{k} c_{k}\right]_{k}^{*}, c_{k+1}\right], \ldots\right], c_{n}\right], c_{n+1}\right]\right.
\end{gathered}
$$

holds for any choice of the elements $r_{i} \in \mathrm{Z}_{n}(R)$ and $c_{i} \in C(1 \leq i \leq n+1)$. 
Clearly, $r_{n+1} \in \mathrm{Z}_{n}(R)$ implies that

$$
\left[r_{1} c_{1}, \ldots, r_{n} c_{n}, r_{n+1} c_{n+1}\right]_{n+1}^{*}=\left[\left[r_{1} c_{1}, \ldots, r_{n} c_{n}\right]_{n}^{*}, r_{n+1} c_{n+1}\right]=
$$

$r_{n+1}\left[\left[r_{1} c_{1}, \ldots, r_{n} c_{n}\right]_{n}^{*}, c_{n+1}\right]+\left[\left[r_{1} c_{1}, \ldots, r_{n} c_{n}\right]_{n}^{*}, r_{n+1}\right] c_{n+1}=r_{n+1}\left[\left[r_{1} c_{1}, \ldots, r_{n} c_{n}\right]_{n}^{*}, c_{n+1}\right]$ proving our claim for $k=n$.

In view of the commutativity of $C$ we have $\left[x c, c^{\prime}\right]=\left[x, c^{\prime}\right] c$ for all $x \in R$ and $c, c^{\prime} \in C$, whence

$$
r_{n+1}\left[r_{n}\left[\ldots\left[r_{k+1}\left[x c_{k}, c_{k+1}\right], \ldots\right], c_{n}\right], c_{n+1}\right]=r_{n+1}\left[r_{n}\left[\ldots\left[r_{k+1}\left[x, c_{k+1}\right], \ldots\right], c_{n}\right], c_{n+1}\right] c_{k}
$$

follows. Now assume that our claim holds for some $2 \leq k \leq n$, then we obtain that

$$
\begin{gathered}
{\left[r_{1} c_{1}, \ldots, r_{n} c_{n}, r_{n+1} c_{n+1}\right]_{n+1}^{*}=} \\
r_{n+1}\left[r_{n}\left[\ldots\left[r_{k+1}\left[\left[r_{1} c_{1}, \ldots, r_{k} c_{k}\right]_{k}^{*}, c_{k+1}\right], \ldots\right], c_{n}\right], c_{n+1}\right]= \\
r_{n+1}\left[r_{n}\left[\ldots\left[r_{k+1}\left[\left[\left[r_{1} c_{1}, \ldots, r_{k-1} c_{k-1}\right]_{k-1}^{*}, r_{k} c_{k}\right], c_{k+1}\right], \ldots\right], c_{n}\right], c_{n+1}\right]= \\
r_{n+1}\left[r_{n}\left[\ldots\left[r_{k+1}\left[r_{k}\left[\left[r_{1} c_{1}, \ldots, r_{k-1} c_{k-1}\right]_{k-1}^{*}, c_{k}\right], c_{k+1}\right], \ldots\right], c_{n}\right], c_{n+1}\right]+ \\
r_{n+1}\left[r_{n}\left[\ldots\left[r_{k+1}\left[\left[\left[r_{1} c_{1}, \ldots, r_{k-1} c_{k-1}\right]_{k-1}^{*}, r_{k}\right] c_{k}, c_{k+1}\right], \ldots\right], c_{n}\right], c_{n+1}\right]= \\
r_{n+1}\left[r_{n}\left[\ldots\left[r_{k+1}\left[r_{k}\left[\left[r_{1} c_{1}, \ldots, r_{k-1} c_{k-1}\right]_{k-1}^{*}, c_{k}\right], c_{k+1}\right], \ldots\right], c_{n}\right], c_{n+1}\right]+ \\
\left.r_{n+1}\left[r_{n}\left[\ldots\left[r_{k+1}\left[\left[\left[r_{1} c_{1}, \ldots, r_{k-1} c_{k-1}\right]\right]_{k-1}^{*}, r_{k}\right] c_{k}, c_{k+1}\right], \ldots\right], c_{n}\right], c_{n+1}\right]= \\
r_{n+1}\left[r_{n}\left[\ldots\left[r_{k+1}\left[r_{k}\left[\left[r_{1} c_{1}, \ldots, r_{k-1} c_{k-1}\right]_{k-1}^{*}, c_{k}\right], c_{k+1}\right], \ldots\right], c_{n}\right], c_{n+1}\right]+ \\
r_{n+1}\left[r_{n}\left[\ldots\left[r_{k+1}\left[\left[\left[r_{1} c_{1}, \ldots, r_{k-1} c_{k-1}\right]_{k-1}^{*}, r_{k}\right], c_{k+1}\right], \ldots\right], c_{n}\right], c_{n+1}\right] c_{k}
\end{gathered}
$$

for all $r_{i} \in \mathrm{Z}_{n}(R)$ and $c_{i} \in C(1 \leq i \leq n+1)$. Since $r_{k} \in \mathrm{Z}_{n}(R)$, the substitution of $c_{k}=1 \in C$ in the above identity gives that

$$
\begin{gathered}
0=\left[r_{1} c_{1}, \ldots, r_{k-1} c_{k-1}, r_{k}, r_{k+1} c_{k+1}, \ldots, r_{n+1} c_{n+1}\right]_{n+1}^{*}= \\
r_{n+1}\left[r_{n}\left[\ldots\left[r_{k+1}\left[r_{k}\left[\left[r_{1} c_{1}, \ldots, r_{k-1} c_{k-1}\right]_{k-1}^{*}, 1\right], c_{k+1}\right], \ldots\right], c_{n}\right], c_{n+1}\right]+ \\
r_{n+1}\left[r_{n}\left[\ldots\left[r_{k+1}\left[\left[\left[r_{1} c_{1}, \ldots, r_{k-1} c_{k-1}\right]_{k-1}^{*}, r_{k}\right], c_{k+1}\right], \ldots\right], c_{n}\right], c_{n+1}\right]= \\
r_{n+1}\left[r_{n}\left[\ldots\left[r_{k+1}\left[\left[\left[r_{1} c_{1}, \ldots, r_{k-1} c_{k-1}\right]_{k-1}^{*}, r_{k}\right], c_{k+1}\right], \ldots\right], c_{n}\right], c_{n+1}\right],
\end{gathered}
$$

whence

$$
\begin{gathered}
{\left[r_{1} c_{1}, \ldots, r_{n} c_{n}, r_{n+1} c_{n+1}\right]_{n+1}^{*}=} \\
r_{n+1}\left[r_{n}\left[\ldots\left[r_{k+1}\left[r_{k}\left[\left[r_{1} c_{1}, \ldots, r_{k-1} c_{k-1}\right]_{k-1}^{*}, c_{k}\right], c_{k+1}\right], \ldots\right], c_{n}\right], c_{n+1}\right]
\end{gathered}
$$

follows. Thus the validity of our claim inherits from $k$ to $k-1$.

For $k=1$ the above claim gives that

$$
\left[r_{1} c_{1}, \ldots, r_{n} c_{n}, r_{n+1} c_{n+1}\right]_{n+1}^{*}=r_{n+1}\left[r_{n}\left[\ldots\left[r_{2}\left[r_{1} c_{1}, c_{2}\right], \ldots\right], c_{n}\right], c_{n+1}\right]
$$

for all $r_{i} \in \mathrm{Z}_{n}(R)$ and $c_{i} \in C(1 \leq i \leq n+1)$. Take $c_{1}=1 \in C$ in the above identity and use $r_{1} \in \mathrm{Z}_{n}(R)$ to derive

$$
0=\left[r_{1}, r_{2} c_{2}, \ldots, r_{n} c_{n}, r_{n+1} c_{n+1}\right]_{n+1}^{*}=r_{n+1}\left[r_{n}\left[\ldots\left[r_{2}\left[r_{1}, c_{2}\right], \ldots\right], c_{n}\right], c_{n+1}\right],
$$

whence

follows.

$$
\begin{gathered}
{\left[r_{1} c_{1}, \ldots, r_{n} c_{n}, r_{n+1} c_{n+1}\right]_{n+1}^{*}=r_{n+1}\left[r_{n}\left[\ldots\left[r_{2}\left[r_{1} c_{1}, c_{2}\right], \ldots\right], c_{n}\right], c_{n+1}\right]=} \\
r_{n+1}\left[r_{n}\left[\ldots\left[r_{2}\left[r_{1}, c_{2}\right], \ldots\right], c_{n}\right], c_{n+1}\right] c_{1}=0
\end{gathered}
$$

\section{ACKNOWLEDGMENT}

The authors sincerely thank the referee for many helpful comments which helped to improve the exposition of the original submitted paper. 


\section{REFERENCES}

(1) [Ch] V. R. Chandran, On two analogues of Cohen's theorem, Pure Appl. Math. Sci. 7(1-2) (1978), 5-10.

(2) [Co] I. S. Cohen, Commutative rings with restricted minimum condition, Duke Math. J. 17 (1950), 27-42.

(3) [D] V. Drensky, Free Algebras and PI-Algebras, Springer-Verlag, New York, 2000.

(4) [DF] V. Drensky and E. Formanek, Polynomial Identity Rings, BirkhäuserVerlag, Basel, 2004.

(5) [H] F. Hansen, On one-sided prime ideals, Pacific J. Math. 58(1) (1975), 79-85.

(6) [Je] S. A. Jennings, On rings whose associated Lie rings are nilpotent, Bull. Amer. Math. Soc. 53 (1947), 593-597.

(7) [Jo] P. Jothilingam, Cohen's theorem and Eakin-Nagata theorem revisited, Comm.. Algebra 28(10) (2000), 4861-4866.

(8) [Ka] I. Kaplansky, Elementary divisors and modules, Trans. Amer. Math. Soc. 66 (1949), 464-491.

(9) [Ko] K. Koh, On prime one-sided ideals, Canad. Math. Bull. 14(2) (1971), 259-260.

(10) $[\mathrm{Kr}] \mathrm{G}$. Krause, On fully left bounded left noetherian rings, J. Algebra 23 (1972), 88-99.

(11) [L] C.-P. Lu, Spectra of modules, Comm. Algebra 23(10) (1995), 37413752.

(12) $[\mathrm{Na}]$ A. R. Naghipour, A simple proof of Cohen's theorem, Amer. Math. Monthly 112(9) (2005), 825-826.

(13) [Ni] I. Nishitani, A Cohen-type theorem for Artinian modules, Arch. Math. 87(3) (2006), 206-210.

(14) [Re] M. L. Reyes, Noncommutative generalizations of theorems of Cohen and Kaplansky, Algebr. Represent. Theory 15(5) (2012), 933-975.

(15) [Ro] L. H. Rowen, Polynomial Identities in Ring Theory, Academic Press, New York, 1980.

(16) [S] R. Y. Sharp, Steps in Commutative Algebra, Second edition, Cambridge University Press, Cambridge, 2000.

(17) [Sz] J. Szigeti, New determinants and the Cayley-Hamilton theorem for matrices over Lie nilpotent rings, Proc. Amer. Math. Soc. 125(8) (1997), 2245-2254.

(18) [SzvW] J. Szigeti and L. van Wyk, Determinants for $n \times n$ matrices and the symmetric Newton formula in the $3 \times 3$ case, Linear and Multilinear Algebra, Published online: 16 Jul 2013, DOI:10.1080/03081087.2013.806919

Institute of Mathematics, University of Miskold, Miskold, Hungary 3515

E-mail address: jeno.szigeti@uni-miskolc.hu

Department of Mathematical Sciences, Stellenbosch University, P/Bag X1, Matieland 7602, Stellenbosch, South Africa

E-mail address: LvW@sun.ac.za 